\title{
Intestinal Parasite Infection and Its Association with Undernutrition among Early Adolescents in Hawassa University Technology Village, Southern Ethiopia
}

\author{
Amelo Bolka Gujo (iD, ${ }^{1}$ Assefa Philipos Kare ${ }^{D},{ }^{1}$ and Shambel Gussa Shuramo \\ ${ }^{1}$ Department of Social and Population Health, Yirgalem Hospital Medical College, Yirgalem, Ethiopia \\ ${ }^{2}$ Tula Sub-City Health Office, Hawassa City Administration, Hawassa, Ethiopia \\ Correspondence should be addressed to Amelo Bolka Gujo; amelobolka@gmail.com
}

Received 13 May 2021; Revised 5 September 2021; Accepted 22 September 2021; Published 15 October 2021

Academic Editor: Swati Sakhuja

Copyright (c) 2021 Amelo Bolka Gujo et al. This is an open access article distributed under the Creative Commons Attribution License, which permits unrestricted use, distribution, and reproduction in any medium, provided the original work is properly cited.

\begin{abstract}
Background. Different studies presented negating findings of the association between intestinal parasite infections (IPIs) and undernutrition among early adolescents in Ethiopia. This study was aimed at assessing intestinal parasite infection and its association with undernutrition among early adolescents in four selected districts of the Sidama region. Method. An institutionbased cross-sectional study was conducted in October 2020 among 792 early adolescents. The multistage stage sampling was applied to select 16 primary schools. Simple random sampling was applied to select study participants. Trained data collectors administered questionnaires. Stool samples were collected and analyzed. Anthropometric measurements were taken and indices were calculated using AnthroPlus software. Data were entered into and analyzed by SPSS version 25 software. Association between IPI and undernutrition was measured using multivariable analysis. The outputs are presented using an adjusted odds ratio (AOR) with 95\% confidence intervals (CIs). Result. The prevalence of IPI, thinness, and stunting was 32\% (95\% CI: $28.7 \%, 35.3 \%$ ), $17.5 \%$ (95\% CI: 14.8\%, 20.2\%), and 21.5\% (95 CI: 18.6\%, 24.4\%), respectively. The higher odds of IPIs were observed among adolescents stunted $(\mathrm{AOR}=3.61 ; 95 \% \mathrm{CI}: 2.44-5.33)$, those who are thin (AOR $=3.07 ; 95 \% \mathrm{CI}: 2.02-4.66)$, those who did not wash their hands after toilet $(\mathrm{AOR}=1.89 ; 95 \% \mathrm{CI}: 1.35-2.66)$, those who ate raw meat $(\mathrm{AOR}=1.50 ; 95 \% \mathrm{CI}: 1.03-2.14)$, and those whose family did not own toilet $(\mathrm{AOR}=1.71 ; 95 \% \mathrm{CI}: 1.18-2.46)$. Conclusion. The prevalence of IPI, thinness, and stunting was high and has public health significance in the study area. IPIs were associated with stunting, thinness, lack of toilets, not washing hands after a toilet visit, and eating raw meat. Strengthening nutrition interventions, deworming programs, and health education on personal and environmental hygiene and sanitation are recommended.
\end{abstract}

\section{Introduction}

Our world is a house of 1.2 billion adolescent population of which $90 \%$ live in low- and middle-income countries [1]. The adolescent is defined as a person aged 10-19 years, which is a period of gradual transition from childhood to adulthood. Early adolescence is the first stage and occurs from ages 10 to 14 years [2]. The adolescence period is a critical period of physical growth and development. It is a period of $15-20 \%$ of adult height, up to $60 \%$ of skeletal mass, and $50 \%$ of adult body weight development [3]. It is the second window of opportunity in terms of nutritional status
[4]. It is a time of increased nutritional needs, and lifelong health and nutrition behaviors are formed [5].

Parasitic infections caused by intestinal helminths and protozoans are among the most prevalent infections in the globe, carrying a high burden of morbidity and mortality [6]. Globally, 2 billion people, majorly children, were infected by soil-transmitted helminths (STHs) and schistosomiasis, of whom more than 300 million suffer from associated severe morbidity [7]. About 550 million primary school adolescents live in areas where these parasites are extensively transmitted. STHs are widely distributed all over the world with the greatest numbers occurring in Sub-Saharan Africa [7]. 
People living in the least developed countries are most vulnerable to intestinal parasitic infections mainly due to poverty and malnutrition [4]. In the case of Sub-Saharan Africa, almost half of the primary school early adolescents were infected with one or more intestinal parasitic worms [8]. Different studies conducted in Ethiopia showed that intestinal parasitic infections were higher among school-age children and early adolescents due to their habits of playing or the handling of infested soil, eating with soiled hands, unhygienic toilet practices, drinking, and eating of contaminated water and foods [9-11].

The Federal Ministry of Health of Ethiopia started the health extension program in 2004 and trained and assigned extension workers at almost every village in rural as well as urban areas to create awareness of prevention methods of intestinal parasites and other infections [12]. Despite efforts, due to climatic conditions, poverty, poor personal hygiene, poor environmental sanitation, and lack of safe and clean water, IPIs have been a major health burden of the country, especially in primary school early adolescents [13]. There is also variation in the prevalence rate of IPI in rural and urban areas. The poorest people living in rural areas and in urban slums are the most affected by IPIs [14].

Intestinal helminths and protozoan infections impact host nutrition through a number of mechanisms that may have additive or multiplicative impacts, especially in school children and early adolescents. It causes and/or aggravates undernutrition through worm-induced gastrointestinal tract pathophysiology and food intake, chronic blood loss, and intestinal inflammation, which disturb the absorption of nutrients from the gut [15].

In early adolescents, undernutrition caused by helminth infections/low food intake has been a major public health problem but largely ignored as a target of public health and nutrition programs [16]. In addition to IPIs, in Ethiopia, the inequity between male and female adolescents (due to cultural influence, females are nutritionally vulnerable consuming less nutrients than their fair share relative to males) increased the risk for poor nutrition and health in girls [17]. The actual number of stunted and underweight adolescents has risen in Ethiopia due to rapid population growth [18].

Childhood undernutrition may reduce early adolescent immune function, impairing the body's resistance to infectious diseases and increasing the risk of school absenteeism and drop-out rates [19]. Its long-term consequences are associated with impaired cognitive development and poor school achievement, growth retardation, reduced economic productivity, and poor reproductive health outcomes in females. It also increases the risk for nutritionrelated chronic diseases in adulthood age [20].

Several studies conducted in Ethiopia assessed the prevalence of IPIs in adolescents but failed to associate with undernutrition [11, 21-26]. There is also limited evidence about the magnitude of undernutrition among early adolescents in the study area. Therefore, this study was carried out to identify the recent burden of intestinal parasitic infection and its association with undernutrition among primary school, early adolescents in selected districts of
Hawassa University Technology Village in Sidama National Regional State.

\section{Methods}

2.1. The Study Area. This study was conducted in 16 primary schools found in four randomly selected districts out of seven districts located in Hawassa University Technology Village, Sidama National Regional State. Boricha, Loka Abaya, Daara, and Bona were selected districts located at $311 \mathrm{~km}, 342 \mathrm{~km}, 260 \mathrm{~km}$, and $385 \mathrm{~km}$ south of Addis Ababa, respectively, the capital of Ethiopia. The selected districts have 192 primary schools and 233,228 primary school children. The physical health service coverage of the village was $100 \%$. The village has two general and two primary level hospitals. Agriculture is the main source of income on the site. More than $85 \%$ of the inhabitants of the village's livelihood depend on farming. Major crops grown in the village include Enset (false banana), cereals, cash crops (khat and coffee), and livestock.

2.1.1. Source Population. The source population of the study was all (10-14 years) early adolescents enrolled in primary schools of the selected districts.

2.1.2. Study Population. The study population was all selected early adolescents registered for the academic year of 2020 , in sixteen primary schools of the selected districts.

2.1.3. Study Design. A facility-based analytical cross-sectional study was carried out in the Hawassa University Technology Village in October 2020.

2.1.4. Inclusion Criteria. All healthy early adolescents aged 10-14 years who had parental consent to participate in research from the selected primary schools were included.

2.1.5. Exclusion Criteria. All students who had a known history of deworming tablet consumption in the previous six months/IP infection, were seriously ill, and were physically disabled were excluded from the study.

2.1.6. Sample Size Calculation. The sample sizes for each specific objective were calculated using a single population proportion formula taking the assumptions of 5\% margin of error and 95\% confidence level. From previous studies, the prevalence of $20.7 \%$ for stunting [9], 27.5\% for thinness [9], and $62.4 \%$ for IPI [26] were used. Using the design effect of two and $10 \%$ of nonresponse rate, sample sizes of 556, 674, and 792 were calculated for the prevalence of stunting, thinness, and IP infection, respectively. A sample size of 792 obtained from the IP infection was used because it was the largest sample size estimated and would be sufficient for the study. 
2.1.7. Sampling Procedure. The multistage stage sampling was applied to select 16 primary schools located in Hawassa University Technology Village, Sidama National Regional State. At the first stage, four districts were randomly selected out of seven. In the second stage, four primary schools were also randomly selected from each selected district. Simple random sampling was applied to select 792 study participants. The calculated sample size was proportionally allocated to each school according to the number of students.

2.1.8. Data Collection Process and Quality Assurance. A structured and interviewer-administered questionnaire was used. The questionnaire was adapted from different related works of the literature $[14,27,28]$. It was first prepared in English and translated into Sidaamu Afoo (local language) and back-translated to the English language by language experts for data collection. Before the data collection, five percent of the sample size was pretested to check clarity and local understanding of the points included in the data collection tool. Eight degree holder nurses and four laboratory technologists for data collection and four public health experts for supervision were recruited and trained for 3 days before the data collection. The data were collected by developing three teams; each team had three data collectors and a supervisor. The principal investigators were also involved in the field supervision to ensure the overall quality of the data.

2.2. Weight. Early adolescents were weighed using Seca digital scales which were validated against standard weights before taking the actual measurements. The scales were placed on a hard, flat surface. Early adolescents wearing only lightweight clothing, excluding shoes, belts, socks, watches, jackets, and heavy items from the pocket, were weighted. Measurement was taken twice, and an average of the two was used for analysis. Based on the 2007 World Health Organization standard reference values, the body mass index for ages below $<2$ Z-score was defined as thin [28].

2.3. Height. The measurement of height was done on a vertical wall with an attached measuring tape and a horizontal headboard that could be brought into contact with the uppermost point on the head. The moveable headboard was brought on to the topmost point on the head with sufficient pressure to compress the hairs. The height is measured in meters and recorded to the nearest $0.1 \mathrm{~cm}$. The height was measured barefoot or in thin socks. Measurement was taken twice, and an average of the two was used for analysis. Based on the 2007 WHO standard reference values, height for age Z-score below $<2 \mathrm{Z}$-score was defined as stunted [28].

2.4. Stool Examination. After informing how to bring the stool specimen a clean, stool specimen container was given with an applicator stick to each study participant. At the time of collection, the date of sampling, school name, the name of the participant, age, and sex were recorded for each subject in a recording format. About $2 \mathrm{~g}$ of stool specimens was collected from each student and mixed with $10 \%$ formalin for preservation. The preserved fecal specimens had been transported to the laboratory of the Leku hospital. All specimens were processed by using formal ether fecal concentration techniques as indicated in the WHO standard operating procedures for the parasitological examination of feces [29]. The direct microscopy method was applied to identify intestinal parasites.

A senior laboratory technician of the hospital reprocessed $10 \%$ of randomly selected fecal samples, and the results were compared with the results made by the original laboratory technician.

2.4.1. Data Management and Analysis. Data were cleaned, coded, and entered into, and analyzed by SPSS version 25 software. The AnthroPlus software was used to calculate anthropometric indices. Binary logistic regression was employed to determine the odds ratio for bivariable analysis. Candidate variables with $P \leq 0.2$ were selected for multivariable logistic regression analysis. The outputs are presented using an adjusted odds ratio with 95\% confidence intervals. Statistical significance was declared at $P \leq 0.05$.

2.4.2. Ethical Consideration. Ethical clearance was obtained from the Institutional Review Board (IRB) of College of Medicine and Health Science, Hawassa University (Ref. No: IRB/216/13; Date 17/9/2020). A formal letter was written from the School of Nutrition, Food Science and Technology to selected districts' education offices. Concerned officials were informed about the purpose of the study, and a permission letter was taken. Data were collected after taking informed consent from the parents/caretakers. Study participants were told the laboratory analysis and physical examination results. Personal hygiene/sanitation and nutrition counseling was provided. Positive cases for IPIs and undernourished were consulted and linked to local health centers and hospitals for the treatment.

\section{Result}

3.1. Sociodemographic Characteristics of the Respondents/ Family. A total of seven hundred fifty early adolescents in secondary cycle primary school were enrolled in the study with an overall response rate of $94.7 \%$. The mean ( \pm standard deviation) age of the early adolescents was $12.4( \pm 1.60)$ years. Four hundred seventy-eight $(63.7 \%)$ of the respondents were older than or equal to 12 years, whereas three hundred ninety-five $(52.7 \%)$ were females. Concerning the ethnicity of respondents, 691 (92.1\%) were Sidama. Almost all, 710 (94.7\%), adolescents' mothers/ caregivers were married. Slightly greater than two-thirds, 533(71\%), of the study participants' families were Protestant religion followers. Pertaining to the place of residence, nine in ten $(90.3 \%)$ were rural dwellers. About two-thirds of the respondents $(65.5 \%)$ had five or more family members (Table 1). 
TABLE 1: Sociodemographic characteristics of primary school early adolescents in selected districts of Hawassa University Technology Village, Southern Ethiopia, October 2020.

\begin{tabular}{lccc}
\hline Variable $(n=750)$ & Category & Frequency & $\begin{array}{c}\text { Percent } \\
(\%)\end{array}$ \\
\hline \multirow{2}{*}{ Age of adolescent } & $<12$ years & 272 & 36.3 \\
& $\geq 12$ years & 478 & 63.7 \\
\hline \multirow{2}{*}{ Sex of adolescent } & Male & 355 & 47.3 \\
& Female & 395 & 52.7 \\
\hline \multirow{2}{*}{ Residence } & Rural & 677 & 90.3 \\
& Urban & 73 & 9.7 \\
\hline \multirow{2}{*}{ Ethnicity } & Sidama & 691 & 92.1 \\
& Oromo & 39 & 5.2 \\
& Amhara & 20 & 2.7 \\
\hline \multirow{2}{*}{ Religion } & Protestant & 533 & 71 \\
& Orthodox & 140 & 18.7 \\
\multirow{2}{*}{ Marital status of mothers/ } & Muslim & 77 & 10.3 \\
caretakers & Married & 710 & 94.7 \\
& Divorced & 22 & 2.9 \\
\multirow{2}{*}{ Number of family members } & Widowed & 18 & 2.4 \\
\hline
\end{tabular}

3.1.1. Socioeconomic Characteristics of the Respondents/ Family. Pertaining to the educational status of the study participants' parents, nearly half, $362(48.3 \%)$ of mothers and $230(30.7 \%)$ of fathers were not attended formal education. Concerning the occupation, two-thirds (67.9\%) of fathers were farmers whereas six in ten $(60.4 \%)$ of the mothers were housewives. The vast majority of respondents, 653(87.1\%) families earned monthly income less than or equal to 2000 Ethiopian Birr (Table 2).

3.1.2. Water, Sanitation, and Hygiene (WASH) Practices of Respondents' Families. This study revealed that 276 (36.8\%) of the study participants' families lacked toilet facilities. About one-fourth, 201 (26.8\%), of the respondents' families had a history of sharing the toilet with other families or neighbors. Slightly more than one-third, $265(35.3 \%)$, of the respondents' families disposed waste in the open field. Piped outside the compound was the source of water for $618(82.4 \%)$ of the families of the study participants. Ninety-seven (12.9\%) reported that they walked on foot for more than thirty minutes to fetch water. About onequarter, $197(26.3 \%)$, used some methods of water purification (Table 3).

\subsubsection{Personal Hygiene Practices of Study Participants.} Of the total respondents, three hundred thirty-six (44.8\%) reported that they did not wash their hands after a toilet visit. Dirty in the fingernails was observed in nearly half, 360 (48\%), of respondents. Concerning handwashing with soap, $405(54 \%)$ of the early adolescents used soap for washing their hands. Of the 750 respondents, 185 (24.7\%) did not wear shoes. One-third, 255(34\%), had a history of raw meat/vegetable consumption at least once a week (Table 4).
3.1.4. Nutritional Status of Early Adolescents. In this study, we investigated undernutrition among early adolescents using two indicators: stunting and thinness. The mean ( \pm standard deviation) age-specific $Z$-score for BMI among early adolescents was $-0.42( \pm 0.79)$. The prevalence of thinness was $17.5 \%$ (95\% CI: $14.8 \%, 20.2 \%)$. Of all respondents, $110(14.7 \%)$ and $21(2.8 \%)$ were observed with moderate and severe thinness, respectively. The mean $( \pm \mathrm{SD})$ HAZ-score of the respondents was $-1.06( \pm 0.92)$. The prevalence of stunting was $21.5 \%$ (95 CI: $18.6 \%, 24.4 \%$ ). Of all study participants, $133(17.8 \%)$ and $28(3.7 \%)$ were diagnosed with moderate and severe stunting, respectively.

\subsubsection{Prevalence of IP Infections among Early Adolescents.} This study revealed that 32\% (95\% CI: $28.7 \%, 35.3 \%$ ) of respondents were infected by intestinal parasites. Of those infected by IP, 227 (98.4\%) were infected by one parasite only (monoparasitism), whereas the remaining had polyparasitism. The most prevalent IP infection identified was Ascaris lumbricoides (A. lumbricoides) 138 (18.4\%) followed by Trichuris trichiura (T. trichiura) 63 (8.4\%), Schistosoma mansoni (S. mansoni) 22(2.9\%), and Giardia lamblia (G. lamblia) 4 (0.7\%) (Figure 1).

3.1.6. Factors Associated with IPIs. The odds of IPIs were 3.6 times higher among stunted adolescents compared with not stunted (AOR $=3.61 ; 95 \%$ CI: 2.44-5.33). Thin adolescents were three times more likely to have intestinal parasite infections than their counterparts $(\mathrm{AOR}=3.07 ; 95 \% \mathrm{CI}$ : 2.02-4.66). Compared with those who had no history of eating raw meat at least per week, adolescents who ate raw meat once per week had 1.5 times higher odds of being infected by intestinal parasites $(\mathrm{AOR}=1.50 ; 95 \%$ CI: $1.03-2.14)$.

Early adolescents from families who not owning toilet facilities were 1.7 times more likely to be infected by intestinal parasites compared to their counterparts $(\mathrm{AOR}=1.71 ; 95 \% \mathrm{CI}: 1.18-2.46)$. Similarly, the odds of IPIs were $1.89(\mathrm{AOR}=1.89 ; 95 \% \mathrm{CI}: 1.35-2.66)$ times higher among early adolescents who did not wash their hands after the toilet visit compared to early adolescents who washed their hands after the toilet visit (Table 5).

\section{Discussion}

Intestinal parasite infection continued as major threats to health in the least developed countries. Our study investigated the IPIs and their association with undernutrition among early adolescents in selected districts of Hawassa University Technology Village, Sidama Region. The key findings of this study were as follows: $32 \%, 21.5 \%$, and $17.5 \%$ of early adolescents were infected with intestinal parasites, stunted, and wasted, respectively. Stunting, wasting, raw meat consumption history, handwashing practices after toilet, and toilet owning were variables that showed a statistically significant association with IPIs.

This study revealed the $32 \%$ prevalence of IPIs among early adolescents showing its public health burden. This 
TABLE 2: Socioeconomic characteristics of primary school early adolescents in selected districts of Hawassa University Technology Village, Southern Ethiopia, October 2020.

\begin{tabular}{|c|c|c|c|}
\hline Variable $(n=750)$ & Category & Frequency & Percent (\%) \\
\hline \multirow{3}{*}{ Mother education } & No formal education & 362 & 48.3 \\
\hline & Primary level & 213 & 28.4 \\
\hline & Secondary level and above & 175 & 23.3 \\
\hline \multirow{3}{*}{ Father education } & No formal education & 230 & 30.7 \\
\hline & Primary level & 281 & 37.5 \\
\hline & Secondary level and above & 239 & 31.8 \\
\hline \multirow{3}{*}{ Mother occupation } & Housewife & 453 & 60.4 \\
\hline & Merchant & 192 & 25.6 \\
\hline & Employed & 105 & 14 \\
\hline \multirow{3}{*}{ Father occupation } & Farmer & 509 & 67.9 \\
\hline & Merchant & 191 & 25.5 \\
\hline & Employed & 50 & 6.6 \\
\hline \multirow{2}{*}{ Monthly income level } & $\leq 2000$ Eth Birr & 653 & 87.1 \\
\hline & $>2000$ Eth Birr & 97 & 12.9 \\
\hline
\end{tabular}

TABLE 3: WASH practice of early adolescents in four selected districts of Hawassa University Technology Village, Southern Ethiopia, October, 2020.

\begin{tabular}{|c|c|c|c|}
\hline Variable $(n=750)$ & Category & Frequency & Percent (\%) \\
\hline \multirow{2}{*}{ Family own toilet } & Yes & 474 & 63.2 \\
\hline & No & 276 & 36.8 \\
\hline \multirow{2}{*}{ The history of toilet sharing } & Yes & 201 & 26.8 \\
\hline & No & 549 & 73.2 \\
\hline \multirow{4}{*}{ Sour of water for the family } & Piped into compound & 14 & 1.9 \\
\hline & Piped outside the compound & 618 & 82.4 \\
\hline & Protected well/spring & 57 & 7.6 \\
\hline & Unprotected well/spring & 61 & 8.1 \\
\hline \multirow{2}{*}{ Distance to get the water } & $>30$ minutes & 97 & 12.9 \\
\hline & $\leq 30$ minutes & 653 & 87.1 \\
\hline \multirow{2}{*}{ Use some methods of water purification } & Yes & 197 & 26.3 \\
\hline & No & 553 & 73.7 \\
\hline \multirow{3}{*}{ Waste disposal system } & In pit & 340 & 45.3 \\
\hline & Burning & 145 & 19.3 \\
\hline & Open field & 265 & 35.3 \\
\hline
\end{tabular}

TAвLE 4: Personal hygiene practices of early adolescents in four selected districts of Hawassa University Technology Village, Southern Ethiopia, October 2020.

\begin{tabular}{|c|c|c|c|}
\hline Variable $(n=750)$ & Category & Frequency & Percent (\%) \\
\hline \multirow{2}{*}{ Wash hand after toilet } & Yes & 414 & 55.2 \\
\hline & No & 336 & 44.8 \\
\hline \multirow{2}{*}{ Use soap for hand wash } & Yes & 405 & 54 \\
\hline & No & 345 & 46 \\
\hline \multirow{2}{*}{ Dirty in fingernails (observed) } & Yes & 360 & 48 \\
\hline & No & 390 & 52 \\
\hline \multirow{2}{*}{ Shoes wearing } & Yes & 565 & 75.3 \\
\hline & No & 185 & 24.7 \\
\hline \multirow{2}{*}{ Consumed raw meat/vegetable in the last week } & Yes & 255 & 34 \\
\hline & No & 495 & 66 \\
\hline
\end{tabular}

finding was consistent with other studies from Ethiopia [30] and Brazil [31]. The present study presented a lower prevalence of IPI compared to previous studies conducted in different parts of Ethiopia [11, 21-23, 25-27], India (36\%) [32], Nigeria (78.1\%) [33], Argentina (78.1\%) [34], Sudan (87.2\%) [24], and Colombia (100\%) [35]. These differences 


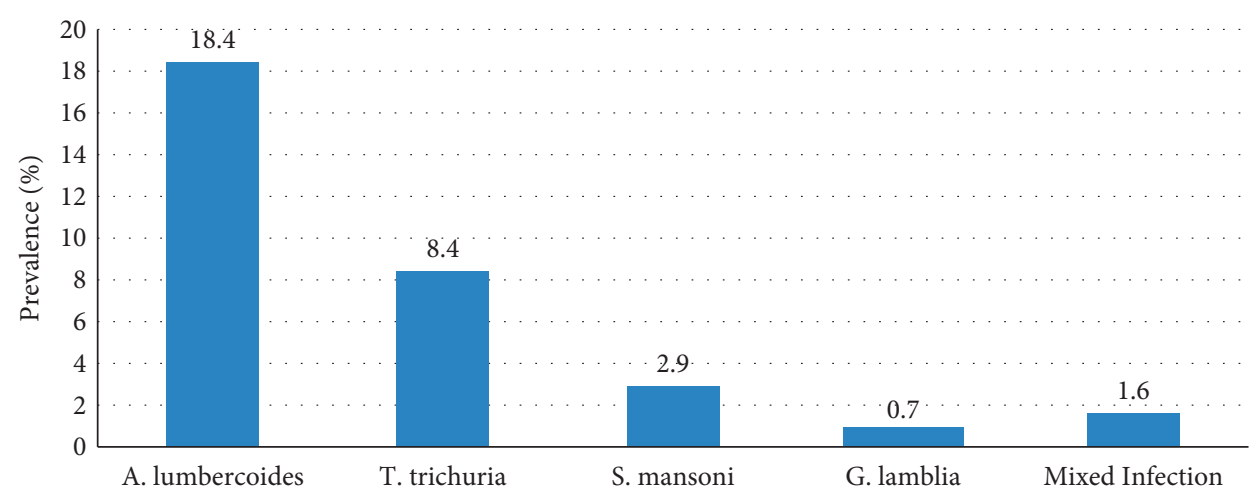

Figure 1: Prevalence of intestinal parasites (species) infection among early adolescents in four selected districts of Hawassa University Technology Village, Southern Ethiopia, October 2020.

TABLE 5: Factors associated with IPIs among primary school early adolescents in the districts of Hawassa University Technology Village, Southern Ethiopia, October 2020.

\begin{tabular}{|c|c|c|c|c|c|c|}
\hline \multirow{2}{*}{ Variable $(n=750)$} & \multirow{2}{*}{ Category } & \multicolumn{2}{|c|}{ IPIs } & \multirow{2}{*}{ COR } & \multirow{2}{*}{ AOR } & \multirow{2}{*}{$P$ value } \\
\hline & & Yes & No & & & \\
\hline \multirow{2}{*}{ Place of residence } & Rural & 206 & 471 & $1.99(1.22-3.25)$ & $1.55(0.89-2.69)$ & \multirow{2}{*}{0.122} \\
\hline & Urban & 34 & 39 & 1 & 1 & \\
\hline \multirow{2}{*}{ Family income level } & $\leq 2000$ eth birr & 201 & 452 & $0.66(0.43-1.03)$ & $0.67(0.42-1.09)$ & \multirow{2}{*}{0.113} \\
\hline & $>2000$ eth birr & 39 & 58 & 1 & 1 & \\
\hline \multirow{2}{*}{ Family own toilet } & Yes & 106 & 170 & 1 & 1 & \multirow{2}{*}{0.004} \\
\hline & No & 134 & 340 & $1.58(1.15-2.17)$ & $1.71(1.18-2.46)$ & \\
\hline \multirow{2}{*}{ Family toilet sharing history } & Yes & 61 & 140 & 1 & 1 & \multirow{2}{*}{0.144} \\
\hline & No & 179 & 370 & $1.11(0.78-1.57)$ & $1.36(0.48-1.11)$ & \\
\hline \multirow{2}{*}{ Sex of the adolescents } & Male & 133 & 262 & 1 & 1 & \multirow{2}{*}{0.087} \\
\hline & Female & 107 & 248 & $1.18(0.86-1.60)$ & $0.73(0.52-1.05)$ & \\
\hline \multirow{2}{*}{ Shoes wearing } & Yes & 177 & 388 & 1 & 1 & \multirow{2}{*}{0.217} \\
\hline & No & 63 & 122 & $1.13(0.79-1.61)$ & $0.78(0.53-1.16)$ & \\
\hline \multirow{2}{*}{ Dirty in adolescents finger nail } & Yes & 134 & 226 & 1 & 1 & \multirow{2}{*}{0.131} \\
\hline & No & 106 & 284 & $1.59(1.17-2.16)$ & $1.30(0.93-1.83)$ & \\
\hline \multirow{2}{*}{ Wash hands after toilet } & Yes & 107 & 307 & 1 & 1 & \multirow{2}{*}{$<0.001$} \\
\hline & No & 133 & 203 & $1.88(1.38-2.56)$ & $1.89(1.35-2.66)$ & \\
\hline \multirow{2}{*}{ Raw meat eating history } & Yes & 94 & 162 & $1.38(1.01-1.90)$ & $1.50(1.03-2.14)$ & \multirow{2}{*}{0.037} \\
\hline & No & 146 & 348 & 1 & 1 & \\
\hline \multirow{2}{*}{ Stunting } & Yes & 89 & 72 & $3.58(2.49-5.15)$ & $3.61(2.44-5.33)$ & \multirow{2}{*}{$<0.001$} \\
\hline & No & 151 & 438 & 1 & 1 & \\
\hline \multirow{2}{*}{ Thinness } & Yes & 71 & 60 & $3.15(2.14-4.64)$ & $3.07(2.02-4.66)$ & \multirow{2}{*}{$<0.001$} \\
\hline & No & 169 & 450 & 1 & 1 & \\
\hline
\end{tabular}

NB: all the variables entered into the multivariable analysis model were also listed under column 1 of Table 5 .

may be explained by the difference in socioeconomic status, time of the study, level of environmental sanitation, lack of access to drinking water, personal hygiene, and methods used in the study.

Assessing the nutritional status of adolescents is essential to improve their health. The prevalence of stunting was $21.5 \%$. This finding was in line with studies conducted in different parts of Ethiopia [9, 23, 36]. In contrast to our study, studies conducted at Adwa [37], Angolela [30], and Wollo [38] reported a lower prevalence of stunting whereas a higher prevalence of stunting was reported from Ethiopia $[39,40]$ and Bangladesh (46.6\%) [41]. These differences in the prevalence of stunting show prolonged food shortage and recurrent infections (IPIs and others) in the target population. Recurrent and prolonged IPIs in adolescents might aggravate stunting by disturbing gastrointestinal tract pathophysiology, food intake, and absorption of nutrients from the gut in which intern affects physiological growth. The difference may be also explained by the difference in socioeconomic status, time of the study, and methods used in the study.

The prevalence of thinness (17.5\%) in the present study was comparable with findings reported from Angolela, Ethiopia [30]. Compared with studies conducted in Ethiopia 
[27, 37, 39], the Philippines [42], and Bangladesh [41], the prevalence of thinness in the present study was low. In contrast to our findings, a low prevalence of thinness was also reported from Ethiopia [23, 36] and Argentina [34]. These reported differences of thinness could be associated with variation in the socioeconomic level, access to food of the family of the target students, time of the study, and methods used in the study.

Increased prevalence of IPIs was identified in adolescents who were stunted. This finding was in line with studies investigated in Ethiopia [23, 30] and Philippines [42]. Similarly, the thinness of the early adolescent was significantly associated with intestinal parasitic infection. The consistent finding was reported by studies conducted in Angolela, Ethiopia [30], and Bahir Dar, Ethiopia [27].

The habit of consuming raw meat among early adolescents increased the odds of intestinal parasite infection. Adolescents who had a habit of at least one-time consumption of raw meat had 1.5 times increased odds of being infected by intestinal parasites. This finding was comparable with studies done in Northwest Ethiopia [25] and Gondar [43]. This could have happened because most of the tapeworms that affect humans come from eating undercooked animal products/meat as well as raw or undercooked fish that is contaminated.

Our study showed that study participants whose families owned the toilet and washed their hands after the toilet visit had a lower chance of being infected by intestinal parasites compared to their counterparts. This finding is consistent with a study conducted in Ethiopia [23, 25, 26], India [32], and Brazil [31]. This is explained as poor personal hygiene and poor environmental sanitation exposed adolescents to IP infections. Specifically, lack of access to toilet facilities for the safe disposal of human waste can result in intestinal parasites and diseases.

\section{Limitation of the Study}

As a limitation, due to the shortage of resources, we applied formal ether fecal concentration techniques with the direct microscopy method which has lower sensitivity to show protozoan species compared to the polymerase chain reaction- (PCR-) based techniques. We recommend researchers should conduct further studies using microscopic examination with PCR assay to increase the possibility of the presence or absence of the infection.

\section{Conclusion}

Our study showed that early adolescents in selected districts of Hawassa Technology village were infected with IPIs, indicating that IPIs continued to be major public health problems in disadvantaged communities. A. lumbricoides was the most predominant intestinal parasite identified. Similarly, the prevalence of thinness and stunting was higher and has public health significance in the study area. Being stunted, being thin, eating raw meat, not owning a toilet, and not washing hands after the toilet were the most important identified risk factors for intestinal parasite infection.
Integrated intervention approaches involving decisionmakers, health professionals, teachers, and communities are crucial. Strengthening nutrition counseling and interventions, school and community deworming programs, health education about personal and environmental hygiene, and sanitation are recommended. Coordination of these efforts is likely to yield appreciable and sustainable gains in improving the health and welfare of early adolescents and securing a prosperous future.
Abbreviations
AOR: Adjusted odds ratio
BMI: Body mass index
CI: $\quad$ Confidence intervals
COR: Crude odds ratio
EDHS: Ethiopia demographic and health survey
HAZ: Height for age Z-score
IPI: Intestinal parasite infection
IRB: Institutional review board
SD: $\quad$ Standard deviation
STH: Soil-transmitted helminths
WASH: Water, sanitation, and hygiene
WHO: World Health Organization.

\section{Data Availability}

The data used in this study are available upon request from the corresponding author.

\section{Conflicts of Interest}

All the authors have declared that they have no conflicts of interest.

\section{Authors' Contributions}

$\mathrm{AB}$ and $\mathrm{SG}$ conceptualized the study; $\mathrm{AB}$ and $\mathrm{AP}$ performed data curation; $A B$ and $S G$ performed formal analysis; $S G$ performed investigation; $\mathrm{AB}$ and $\mathrm{AP}$ developed the methodology; $\mathrm{AB}$ and $\mathrm{AP}$ provided the software; SG supervised the study; $A B$ and $S G$ performed validation; $A B$ wrote the original draft; $\mathrm{AB}, \mathrm{AP}$, and $\mathrm{SG}$ reviewed and edited the article.

\section{Acknowledgments}

The researchers would like to acknowledge Hawassa University College of Medicine and Health Science. Last but not least, the authors also like to acknowledge at heart the staff of Leku General Hospital, study participants, data collectors, and supervisors.

\section{References}

[1] UNICEF Programme, Guidance for the Second Decade: Programming with and for Adolescents, UNICEF. Programme, New York, NY, USA, 2018.

[2] UNICEF Adolescence, An Age of Opportunity; the State of thrE Worls's Children, UNICEF Adolescence, New York, NY, USA, 2011. 
[3] R. K. Benedict, A. Schmale, and S. Namaste, Adolescent Nutrition 2000-2017: DHS Data on Adolescents Age 15-19, DHS Comparative Report No. 47, Rockville, Maryland, USA, 2018.

[4] D. A. P. Bundy, Nd Silva, S. Horton, D. T. Jamison, and G. C. Patto, "Child and adolescent health and development," International Bank for Reconstruction and Development/The World Bank Disease Control Priorities, vol. 8, 3 edition, 2017.

[5] G. C. Patton, S. M. Sawyer, J. S. Santelli et al., "Our future: a Lancet commission on adolescent health and wellbeing," The Lancet, vol. 387, no. 10036, pp. 2423-2478, 2016.

[6] Guideline, Preventive Chemotherapy to Control Soil-Transmitted Helminth Infections in At-Risk Population Groups, World Health Organization, Geneva, Switzerland, Licence: CC BY-NC-SA 3.0 IGO. 2017.

[7] World Health Organization, Helminth Control in School-Age Children. A Guide for Managers of Control Programmes, World Health Organization, Geneva, Switzerland, 2nd edition, 2011

[8] M. O. Harhay, J. Horton, and P. L. Olliaro, "Epidemiology and control of human gastrointestinal parasites in children," Expert Review of Anti-infective Therapy, vol. 8, no. 2, pp. 219-234, 2010.

[9] K. Berhe, A. Kidanemariam, G. Gebremariam, and A. Gebremariam, "Prevalence and associated factors of adolescent undernutrition in Ethiopia: a systematic review and meta-analysis," BMC Nutrition, vol. 49, no. 5, 2019.

[10] L. Chelkeba, Z. Mekonnen, Y. Alemu, and D. Emana, "Epidemiology of intestinal parasitic infections in preschool and school-aged Ethiopian children: a systematic review and meta-analysis," BMC Public Health, vol. 20, no. 1, p. 117, 2020.

[11] T. D. Gebreyesus, T. Tadele, K. Mekete et al., "Prevalence, intensity, and correlates of schistosomiasis and soil-transmitted helminth infections after five rounds of preventive chemotherapy among school children in southern Ethiopia," Pathogens, vol. 9, no. 11,2020

[12] F. A. Desta, G. T. Shifa, D. W. Dagoye, C. Carr, J. V. Roosmalen, and J. Stekelenburg, "Identifying gaps in the practices of rural health extension workers in Ethiopia: a task analysis study," BMC Health Services Research, vol. 839, no. 17, 2017.

[13] N. Fetene, E. Linnander, B. Fekadu, H. Alemu, H. Omer, and M. Canavan, "The Ethiopian health extension program and variation in health systems performance: what matters?" PloS One, vol. 26, 2016.

[14] Central Statistical Agency of Ethiopia, ICF. Ethiopia Demographic and Health Survey. Addis Ababa, Ethiopia, and Rockville, CSA and ICF, Maryland, USA, 2016.

[15] R. Pullan and S. Brooker, "The health impact of polyparasitism in humans: are we under-estimating the burden of parasitic diseases?" Parasitology, vol. 135, no. 7, pp. 783-794, 2008.

[16] P. Christian and E. R. Smith, "Adolescent undernutrition: global burden, physiology, and nutritional risks," Annals of Nutrition and Metabolism, vol. 72, no. 4, pp. 316-328, 2018.

[17] J. Coates, B. N. Patenaude, B. L. Rogers et al., "Intrahousehold nutrient inequity in rural Ethiopia," Food Policy, vol. 81, pp. 82-94, 2018.

[18] Initiatives Development, Global Nutrition Report: Shining a Light to Spur Action on Nutrition, Development Initiatives, Bristol, UK, 2018.

[19] J. E. Mills and O. Cumming, "The impact of water, sanitation and hygiene on key health and social outcomes," UNICEF, vol. 80, 2016.
[20] D. T. Jamison, R. Nugent, H. Gelband, S. Horton, P. Jha, and R. Laxminarayan, "World bank gropu: child and adolescent health and development," UNICEF, vol. 8, 2017.

[21] A. Abossie and M. Seid, "Assessment of the prevalence of intestinal parasitosis and associated risk factors among primary school children in Chencha town, Southern Ethiopia," BMC Public Health, vol. 14, no. 166, p. 166, 2014.

[22] A. Haile, T. Abera, and D. Dana, "The prevalence of intestinal parasitic infection and associated factors among primary school children in gurage zone, south Ethiopia," Journal of Pharmacy and Alternative Medicine, vol. 15, pp. 8-15, 2017.

[23] Z. Mekonnen, D. Hassen, S. Debalke et al., "Soil-transmitted helminth infections and nutritional status of school children in government elementary schools in Jimma Town, Southwestern Ethiopia," SAGE Open Medicine, vol. 8, Article ID 2050312120954696, 10 pages, 2020.

[24] M. N. Mohammed Hamad, A. A. Mokhtar, M. Alameldin, Y. M. Abkar, and M. Eltoum, "Prevalence of intestinal parasitic infections among school aged children in Berber locality, River Nile State, Sudan 2017," Journal of Microbiology \& Experimentation, vol. 7, no. 2, 2019.

[25] B. Sitotaw, H. Mekuriaw, and D. Damtie, "Prevalence of intestinal parasitic infections and associated risk factors among Jawi primary school children, Jawi town, northwest Ethiopia," BMC Infectious Diseases, vol. 341, no. 19, 2019.

[26] B. Sitotaw and W. Shiferaw, "Prevalence of intestinal parasitic infections and associated risk factors among the first-cycle primary schoolchildren in sasiga district, southwest Ethiopia," Journal of parasitology research, vol. 2020, Article ID 8681247, 2020.

[27] T. Hailegebriel, "Undernutrition, intestinal parasitic infection and associated risk factors among selected primary school children in Bahir Dar, Ethiopia," BMC Infectious Diseases, vol. 394, no. 18, 2018.

[28] M. de Onis, A. W. Onyango, E. Borghi, A. Siyam, C. Nishida, and J. Siekmann, "Development of a WHO growth reference for school-aged children and adolescents," Bulletin of the World Health Organization, vol. 85, no. 9, pp. 660-7, 2007.

[29] World Health Organization, Bench Aids for the Diagnosis of Intestinal Parasites, World Health Organization, Geneva, Switzerlandhttps://apps.who.int/iris/handle/10665/37323. 1994.

[30] N. L. Nguyen, B. Gelaye, N. Aboset, A. Kumie, M. A. Williams, and Y. Berhane, "Intestinal parasitic infection and nutritional status among school children in Angolela, Ethiopia," Journal of Preventive Medicine and Hygiene, vol. 53, no. 3, pp. 157-64, 2012.

[31] V. S. Belo, R. B. d. Oliveira, P. C. Fernandes et al., "Fatores associados à ocorrência de parasitoses intestinais em uma população de crianças e adolescentes," Revista Paulista de Pediatria, vol. 30, no. 2, pp. 195-201, 2012.

[32] S. Gopalakrishnan, V. A. Eashwar, M. Muthulakshmi, and A. Geetha, "Intestinal parasitic infestations and anemia among urban female school children in Kancheepuram district, Tamil Nadu," Journal of Family Medicine and Primary Care, vol. 7, no. 6, pp. 1395-400, 2018.

[33] A. Eo, E. Ha, A. Ma, and A. Of, "Prevalence and pattern of intestinal parasites among pupils of private and public primary schools in an urban centre, Nigeria," Nigerian Journal of Paediatrics, vol. 44, no. 2, pp. 56-62, 2017.

[34] M. L. Zonta, P. Cociancic, E. E. Oyhenart, and G. T. Navone, "Intestinal parasitosis, undernutrition and socio-environmental factors in schoolchildren from Clorinda Formosa, Argentina," Revista de Salud Pública, vol. 21, no. 2, pp. 224-231, 2019. 
[35] P. C. Herna'ndez, J. F. Jaramillo, F. Corte's, L. K. Sa'nchez, L. Morales, and J. Chaparro-Olaya, "Intestinal parasitic infections and associated factors in children of three rural schools in Colombia. A cross-sectional study," PloS One, vol. 14, 2019.

[36] Y. M. Demilew and A. A. Emiru, "Under nutrition and associated factors among school adolescents in Dangila Town, Northwest Ethiopia: a cross sectional study," Journal of African Health Sciences, vol. 218, no. 3, p. 18, 2008.

[37] T. Gebregyorgis and T. Tadesse, "Prevalence of thinness and stunting and associated factors among adolescent school girls in Adwa town, north Ethiopia," International Journal of Food Science, vol. 2018, pp. 1-8, 2018.

[38] Y. Menber, D. Tsegaye, A. Woday, H. Cherie, and S. Kebede, "Prevalence of stunting and associated factors among school age children in primary schools of haik town, south Wollo zone, north- eastern Ethiopia, 2017," Journal of Clinical \& Cellular Immunology, vol. 09, no. 01, 2018.

[39] Y. A. Melaku, G. A. Zello, T. K. Gill, R. J. Adams, and Z. Shi, "Prevalence and factors associated with stunting and thinness among adolescent students in Northern Ethiopia: a comparison to World Health Organization standards," Archives of public health $=$ Archives belges de sante publique, vol. 73, no. 44, p. 44, 2015.

[40] E. Z. Tariku, G. A. Abebe, Z. A. Melketsedik, and B. T. Gutema, "Prevalence and factors associated with stunting and thinness among school-age children in arba minch health and demographic surveillance site, southern Ethiopia," PloS One, vol. 13, 2018.

[41] M. A. Rahman, "Prevalence of stunting and thinness among adolesents in rural area of Bangladesh," Journal of Asian Scientific Research, vol. 4, no. 1, pp. 39-46, 2014.

[42] K. Papier, G. M. Williams, R. Luceres-Catubig et al., "Childhood malnutrition and parasitic helminth interactions," Clinical Infectious Diseases, vol. 59, no. 2, pp. 234-243, 2014.

[43] B. Bayleyegn, B. Woldu, A. Yalew, D. Kasew, and F. Asrie, "Prevalence of intestinal parasitic infection and associated factors among HAART initiated children attending at university of gondar comprehensive specialized hospital, northwest Ethiopia," HIV, vol. 13, pp. 81-90, 2021. 